\title{
Chapter 7 \\ Integrated Technological Solutions \\ for Zero Waste Recycling of Printed \\ Circuit Boards (PCBs)
}

\author{
Giacomo Copani, Marcello Colledani, Alessandro Brusaferri, \\ Antonio Pievatolo, Eugenio Amendola, Maurizio Avella and Monica Fabrizio
}

\begin{abstract}
The demand for key metals for the production of high-tech products is constantly growing in Europe, leading to relevant problems both in terms of supply risks and costs. Waste from Electric and Electronic Equipment (WEEE) is growing very fast in Europe, with an annual increase rate between 3 and 5\%. Printed Circuit Boards (PCBs), which are embedded in electric and electronics products, are very valuable waste products, since they are composed also of precious metals and key metals (about 25-30\%). Recycling of PCBs is a very challenging task that has not been solved yet: recycling rates for traditional metals are around 30-35\% and many critical key metals, as well as the non metal fraction, are not recycled. This work proposes a set of solutions to be adopted towards the automated zero-waste treatment of PCBs. They address selective disassembly of PCBs components, mechanical pretreatments, chemical processes for the characterisation of metals material content of PCBs, as well as for the recycling of their non-metal fraction. New business models are finally proposed for the uptake of such solutions in a framework of integrated recycling chain.
\end{abstract}

G. Copani $(\bowtie) \cdot$ M. Colledani · A. Brusaferri

CNR-STIIMA, Istituto di Sistemi e Tecnologie Industriali Intelligenti per il Manifatturiero Avanzato, Milan, Italy

e-mail: giacomo.copani@stiima.cnr.it

A. Pievatolo

CNR-IMATI, Istituto di Matematica Applicata e Tecnologie Informatiche, Milan, Italy

E. Amendola $\cdot$ M. Avella

CNR-IPCB, Istituto dei Polimeri, Compositi e Biomateriali, Pozzuoli, Italy

M. Fabrizio

CNR-ICMATE, Istituto di Chimica della Materia Condensata e di Tecnologie per l'Energia,

Padova, Italy 


\subsection{Scientific and Industrial Motivations}

Due to the globally raising production and consumption of high-tech products, the need of key-metals is constantly increasing. European countries face challenges in terms of supply risks, since they have limited key-metals reserves to be extracted, thus leading to possible negative significant impacts in the manufacturing sector. In 2010, the European Commission identified 14 critical metals for the future of Europe [1]. Extraction of such metals is mainly made in China, Russia, the Democratic Republic of Congo and Brazil, which makes their sourcing affected by political relationships. This situation generates considerable supply risk for Europe, considering that recycling rates are currently limited.

If properly recycled, Waste from Electric and Electronic Equipment (WEEE) represents a strategic source of key-metals. Besides post-use consumer electronics as mobile phones, personal computers, TVs, etc., also waste coming from other sectors using mechatronics product is rich of key-metals (such as automotive, aeronautics, military, industrial automation, etc.). For example, it can be estimated that the content of electronics in a modern vehicle accounts for the $40 \%$ of its entire manufacturing costs [2]. Due to rapid technological trends and to the increased diffusion of electronics, post-consumer WEEE is growing very fast in Europe, with an annual increase rate between 3 and 5\%, which would lead to doubling volumes in the next decade [3]. All WEEE and mechatronics waste include PCBs, which are called urban mineral resources because, on average, they are composed for the $25-30 \%$ of metals such as copper, tin, nickel, gold and silver [4]. Moreover, they contain small quantities of critical metals such as indium, palladium, ruthenium, gallium, tantalum and platinum. Therefore, developing sustainable recycling technologies and systems for PCB key-metals recovery is of fundamental importance for the European recycling and high-tech product manufacturing sectors.

Because of its complexity, recycling of PCBs is a very challenging task that has not been solved yet from the point of view of systems technology and business model sustainability [5]. As a consequence, most of the PCB treatment takes place in China recurring to manual disassembly and processing, leading to negative consequences for human health and environment. Most PCB recycling approaches practiced today, based on pyrolysis and electrolysis, are high energy-demanding, release dioxin or acid drain water in the atmosphere and present significant limitation in the recovery performance. Indeed, only $30-35 \%$ of the PCBs metals can be recovered with achievable purity of $85-95 \%$, depending on the type of material. The remaining materials cannot be economically recycled and recovered, thus they are landfilled. This last fraction includes especially key metals such as tantalum that is present in very low concentrations and is located in specific PCB components (e.g. 35\% in capacitors). Despite the metal part of PCBs, the non-metal fraction, mainly composed of Fibre Reinforced Plastics (FRP), is currently not recycled at all [6].

Smart mechanical pre-treatments coupled with chemical recovery processes would be ideal techniques for addressing the recovery of materials from PCBs (metals and non-metals). In fact, compared to traditional pyro metallurgical processes, 
they involve very limited environmental impact, energy consumption and production of by-products, simultaneously allowing to recover currently landfilled fractions in a logic of zero waste. However, at present, metal recovery rate is still limited and a consistent fraction of precious metals is lost during shredding and separation operations [5]. This constitutes a barrier towards the industrial diffusion of mechanical plants for PCB recycling.

A sustainable PCBs treatment through novel technologies and processes will contribute not only to the solution of the environmental and economic challenge, but will also give the opportunity to increase employment through the establishment of a new advanced industrial recycling sector.

Moving from the outlined limitations and promising technological approaches, this work aims at the definition of a new systemic approach for the sustainable recovery of key-metals and of the non-metallic fraction of (PCBs) in Europe in a zerowaste logic, developing the methodological/technological solutions for supporting its effective use in industry.

The chapter is organised as follows. Section 7.2 presents the state of the art on flexible recycling systems. Section 7.3 outlines the research scope and approach, whereas Sect. 7.4 presents the results of the research. Industrial validation and testing activities are discussed in Sect. 7.5 and, finally, the conclusions together with indications for future research are presented in Sect. 7.6.

\subsection{State of the Art}

State-of-the-art PCB recycling process involves rough dismantling and recovery stages. Disassembly operations are required to remove dangerous components, such as batteries. In general, manual disassembly methods are adopted because of the high degree of variability of post-consumer product streams. Modular disassembly devices can in principle meet the requirements of continuously changing disassembly operations. Moreover, systems integrating manual and automated workstations (hybrid disassembly systems) represent viable solutions. Past research efforts addressed the implementation of semi-automatic solutions for electronic products disassembly. Nonetheless, an integrated solution, capable of processing heterogeneous products such as PCBs is still undeveloped [6]. Besides the limited automation level of the process, disassembly operations are nowadays poorly integrated with the downstream recovery treatments, thus making selective disassembly of highly concentrated components far from being achieved.

The recovery of materials from PCBs includes chemical and physical processes. Among chemical processes, pyrolysis and electrolysis are the most diffused. Pyrolysis enables to obtain friable solid residue from printed circuit boards made of organic, glass fibre and metallic fractions that can be easily separated [7]. The ashes in the residues mainly contain copper, iron, nickel and aluminium that can be further treated by electrolysis and pyro-metallurgical processes [8]. Biotechnology has been outlined as a suitable procedure in metallurgical processing. In particular, in the last 
years, bioleaching was exploited to recover precious metals and copper. However, limited research addressed the bioleaching of metals from WEEE.

The chemical composition of waste must be controlled to select the recovery technologies. Spectrometric techniques are excellent tools available for metal trace and ultratrace analysis of liquid and solid samples. Among them, Inductively Coupled Plasma-Mass Spectrometry (ICP-MS), Laser Ablation-Inductively Coupled PlasmaMass Spectrometry (LA-ICP-MS) and Secondary Ion Mass Spectrometry (SIMS) can provide complete information about chemical composition of waste, but proper analysis procedures have to be developed.

Chemical processes are high energy-demanding and release in the atmosphere dioxin or acid drain water. The alternative is still manual re-usable component disassembly and incineration of the remaining PCB matrix; as a matter of fact, nearly the $80 \%$ of the PCBs collected in the world are processed in China adopting very poor ergonomic and safety conditions [9]. Key-metals, such as tantalum, gallium and indium, that are present in high concentrations only in specific components of PCBs, are currently not recovered and go to landfill [10]. Mechanical treatment represents a promising alternative to pyrolysis with low environmental pollution, limited equipment investments, low energy cost and diversified applications. This treatment consists in mechanical separation and size-reduction to obtain highly concentrated mixtures of metallic and non-metallic fractions. PCBs are an ideal candidate for mechanical shredding and separation but, given the high value of the roughly $30 \%$ metal fraction of a populated circuit board, mechanical shredding and separation systems should be highly efficient to maximize the recovered metals fraction. Detailed physical models estimating the distribution of the size and liberation degree of the output particles, as well as the processing energy required, can pinpoint ideal process operation [5]. Very little research has focused on this area, but stochastic process models, in particular Markov models, are a very promising avenue. Concerning the separation stage, physical models that predict particle trajectories have been developed for eddy current separation and corona electrostatic separation [11]. However, these models consider single trajectories of particles and fail to model two major motivations for the limited separation performance, i.e. particle interactions and impacts, and the presence of unliberated particles in the input material flow. The lack of validated models and control technologies makes comminution and separation processes still uncontrolled in industrial settings. Process parameters are manually set, based on experience coming from trial and error procedures. As a consequence, metal recovery rate is still limited and a consistent fraction of precious metals is lost during shredding and separation [12].

Chemical recycling methods of the Non-Metal Fraction (NMF) in PCBs include gasification, and depolymerisation process using supercritical fluids. The decomposition reactions of epoxy resin in supercritical water (SCW) or using supercritical methanol (SCM) have been studied in literature [13]. The goal of the research in this area is to control the chemical decomposition of organic fraction of PCB to obtain intermediate products suitable for further polymerization reaction. Another promising recycling option is the re-use of the polymer portion as filler in new compounds. Without treatment, these fillers can reduce mechanical properties of the polymers. 
The use of mechano-chemical treatment to prepare scrap-based composites improves the compatibility in blends, since it favours the dispersion of nano-fillers following to the application of high compressive and shear forces.

Finally, from a business model perspective, literature is mainly explorative and takes a comparative country-perspective to illustrate how nations are managing the end-of-life of PCBs and electronic waste [14]. There are currently no clear evidences of consolidated successful business models for PCBs recycling in terms of reverse logistics practices, network supply chain management and companies' vertical integration, infrastructure, secondary markets and pricing strategies. Only some general indications in terms of supply chain actors can be found at waste management system level, but the whole business model with its supporting technologies is not analysed and quantitative performance assessments are lacking.

\subsection{Problem Statement and Research Approach}

From a technological, industrial and business point of view, the main difficulties for the establishment of successful practices for treating PCBs in Europe in a logic of circular economy [15] can be summarised as follows:

- Post-consumer PCBs are a very variable product due to different technologies, design, size and materials composition. This generates uncertainty in the type of waste to be treated. Thus, technology investments and business return are difficult to be forecasted and entrepreneurial risk is implicitly high.

- Limited knowledge is currently available on the elemental composition of PCBmounted components and on the concentrations of key metals in them. Such a situation contributes to increase the above mentioned uncertainty.

- Flexible automation is not generally adopted in treatment processes of PCBs and process control is still poorly deployed in industrial practices: manual practices are the state of the art. This situation poses challenges for safety and health of human operators and, in addition, it makes the treatment non sustainable in Europe, due to the high labour cost compared to emerging countries.

- There is a poor link between the different recycling supply chain processes that are usually carried out by separated companies, in particular between mechanical pre-treatments and downstream recovery. This causes systemic inefficiencies and precious metal fraction losses [16].

- There is a lack of proven successful business models from the economic and environmental point of view. Such a lack discourages entrepreneurs to invest in this promising business application and limits the diffusion of circular economy practices based on PCBs recovery and recycling.

This work aims to address the previous challenges and to overcome the current fragmentation by developing a set of integrated technologies and business enablers in the phases of PCBs disassembly, shredding, separation and materials recycling. In particular, the following innovations are targeted: 
- New knowledge-based selective disassembly technologies for efficient pretreatment of electronic waste. The introduction of automation in operations that are currently manual will lead to a consequent increase of efficiency, safety and quality. Such technologies include: a visual inspection system for the localisation of components to be selectively disassembled on the PCBs; an automated knowledge-based component recognition system based on geometrical and functional analysis of components; an automated system for the characterisation of key-metals in PCBs, based on the elemental composition of the identified components; an automatic selective disassembly process for the extraction of valuable components with the goal of making the downstream mechanical treatment more efficient thanks to a higher concentration of valuable materials to be processed and to the reduction of parts heterogeneity (Sect. 7.4.4).

- Advanced control models and automated technologies for mechanical shredding processes. Knowledge-based models consist in statistical models for predicting the performance of shredding processes in order to optimise the performance of downstream separation processes. Based on these models, automated technologies allow optimal process control adapting parameters to the different type of waste to be processed (Sect. 7.4.1).

- Environmentally-friendly solutions for the recovery and re-use of the metal and non-metal fractions from PCBs. Innovative treatments are needed for recycling the metal and non-metal fraction from PCBs into innovative materials and products to be re-introduced in the market, under a zero-waste perspective. The performance of such solutions is assessed through evaluation and characterization of the physical and mechanical properties of the recycled products and the performance of developed technologies (Sect. 7.4.2).

- New business models for zero-waste treatment of end-of-life PCBs. Existing companies or new entrepreneurial actors will create new supply chains uptaking advanced PCBs recycling technologies to increase overall supply chain business performance (Sect. 7.4.3).

Through the development of the mentioned innovative solutions and business models, this work aims at the establishment of more sustainable PCBs recycling supply chains in Europe, as illustrated in Fig. 7.1. The final goal is to avoid the recourse to heavily impacting pyro-metallurgical processes that require big furnaces and economy of scale, through the establishment of recycling chains, mainly composed of SMEs, which implement highly efficient integrated processes of smart disassembly, mechanical pre-treatment and PCBs materials recovery and re-use.

The research activities reported in this work benefited from a pre-existing pilot plant for the End-Of-Life treatment of PCBs within the facilities of CNR-STIIMA (ex CNR-ITIA) [17]. This pilot plant supports disassembly, shredding and separation processes of mechatronics products embedding PCBs. This infrastructure allowed the testing and validation of developed models and technical innovations on real technologies at disposal of researchers.

Finally, being focused on getting the highest value from PCBs recycled components and materials, the proposed approach is thought to be sustainable even at 

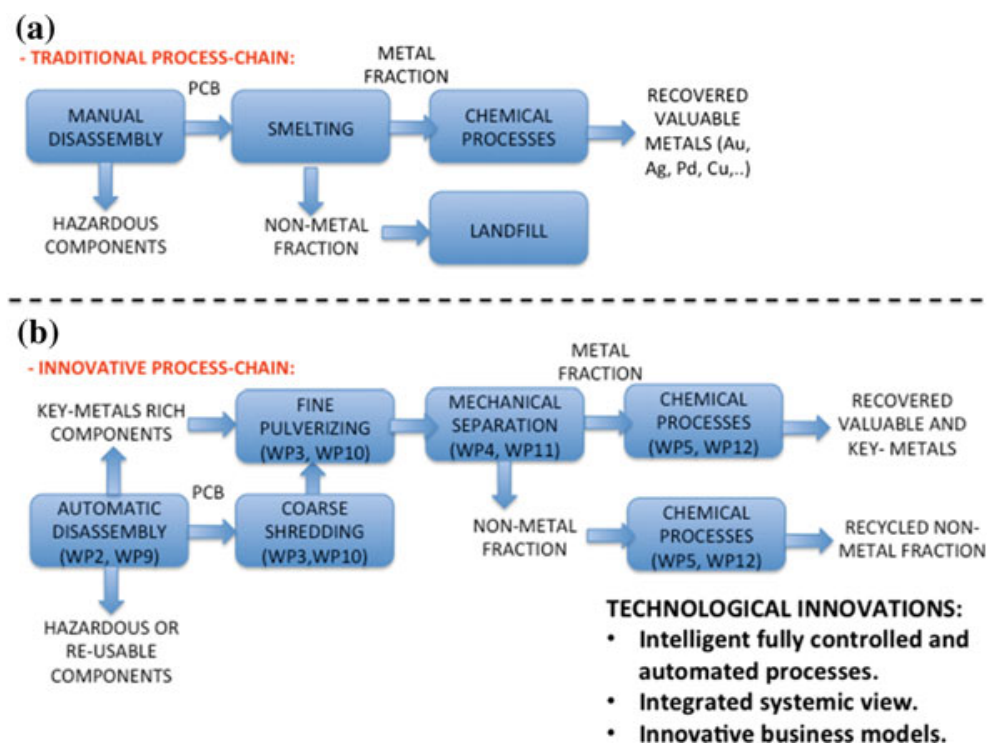

Fig. 7.1 Traditional (a) and innovative (b) PCBs recycling chains

relatively low WEEE collection levels, thus being targeted to SMEs operating in the recycling industry. This makes the results of the research particularly suited for uptake by European recycling industry, which is mainly composed of SMEs.

\subsection{New Solutions for PCBs Recycling}

A set of multi-disciplinary enablers was realised to address the current challenges limiting the recycling of PCBs. Technical solutions were developed to increase the performance of the disassembly and shredding process of PCBs, the assessment of the metal materials composition of shredded PCBs and the recycling of the non-metal fraction. New business models are proposed for the uptake of the developed technologies by supply chain actors. Results are described in the following subsections.

\subsubsection{Shredding Process Modelling and Optimisation}

In a shredding machine, the relationship between input and output mixture has a random nature. In the recycling industry, the operating shredding process parameters are traditionally set based on trial and error procedures and they remain constant regardless of the input mixture. Modelling these kinds of process would allow to set shredding parameters to obtain a desired target mixture out of specific input mate- 
rials, with consequent advantages in time and material saving. For these reasons, a stochastic model has been developed taking into account physical machine parameters. In particular, design and controllable parameters have been considered. The former are the volume of the chamber and the number of the cutting tools (also called knives). The latter are the rotational speed of the rotor and the characteristic size of the output grate (the higher is this value, the largest is the maximum dimension of the particles exiting from the shredder).

The proposed model was built upon the Population Balance Model (PBM) framework [18]. This model study the behavior of particles inside the chamber of a comminution machine by defining three relationships: (1) the proportion of a specified particle type which is selected for breakage per unit of residence time; (2) the degree to which the selected particle type undergoes breakage; (3) the selection of particles which are to be removed from the process. The model predicts the evolution of the particle size and liberation classes during the shredding operations as a function of process parameters.

The transition of mass between size classes in a time interval is governed by a transition matrix $P$ (named Breakage and selection matrix) of proportions that meets the mass conservation constraint. This matrix describes the probability to break a particle from one class to a smaller class. A stochastic dynamic was added to this framework, which is completely deterministic, by allowing the transition matrix itself to be random but centred around $P$. The parameters of the transition probability matrix $P$ are functions of particle breakage and selection probabilities.

As $P$ is unknown, it must be estimated (or trained) from experimental data. A correct quantification of the dispersion of the random matrices around $P$ allowed to decide how many experimental runs were needed to achieve a sufficient precision. This study was first carried out for a batch process and then for a continuous process, which also includes a discharge matrix $D$, different for every grate size. This new matrix determines the fraction of mass in each size class able to leave the comminution chamber in a time interval. From these two matrices a PBM model has been developed, using Markov chains, based onto different assumptions named homogeneity and multiplication. In particular, the first one affirms that $P$ does not depend on time while the second one that the size distribution of shredded particle only depends on the number of breakage intervals.

Based on the random PBM, a procedure for training a model of a specific shredder with a limited number of runs was designed. The trained model can predict the particle size distribution and the output flow in a stationary regime.

\subsubsection{New Technologies to Recycle PCBs Materials}

Recycling of PCBs materials poses multiple challenges considering the current state of the art. Recycling of the Metal Fractions (MF) is affected by uncertainty, since revenues depend by the content of metals of the different type of PBCs. Traditional materials characterisation is not a viable process, since it requires time, it should be 
carried out in certified laboratories and it is expensive. For evaluating the feasibility of MF recovery/re-use, an affordable procedure has been developed for measuring the MF content in PCBs powders obtained as output of mechanical pre-treatments. Literature data $[13,19]$ report mineralization techniques based on acid hydrofluoric (HF) attack. Considering the high danger of this acid, herein a HF-free mineralization method was developed and successfully applied to mechanically processed samples. The development of the HF-free mineralization is very attractive with a view to an industrial application of this method, because HF is a very dangerous acid for health and for the glass part of the spectrometer.

Herein, ICP-MS technique was mainly adopted because this technique is known as a unique tool to evaluate where the elements of interest are present and in which amount, and it is an efficient technique to evaluate their leachability. In fact, the combined use of mass spectrometry techniques (SIMS for surface analysis and ICPMS, LA ICP-MS for bulk analysis) gave useful information about the composition of waste during the various steps of waste processing.

Printed circuit board scraps from different industries have been analysed to estimate the amount of lanthanides and precious metals. It is interesting to remark that ICP-MS results showed that the precious metal content is not correlated to lanthanide amount in the different matrices. For example, PCBs from automotive showed the higher Au and Pd amounts, but the higher Rare Earth content was found in home appliances grinded PCBs (in particular $\mathrm{La}, \mathrm{Ce}$ and $\mathrm{Nd}$ ).

Considering the Non Metal Fraction (NMF) of PCBs, new chemical compounds and new secondary applications should be defined to increase recycling practices. In this work, selective cleavage reactions have been defined for the production of small molecular weight fractions, to be used for the production of new reactive materials. PCBs motherboards are mainly made of an unsaturated polyester resin (UPR). The presence of reversible chemical linkages, such as esters, was exploited to split the thermosetting materials into smaller fragments that are suitable to further chemical processing. Although the direct polymerization reaction to produce UPR involves esterification by condensation of acids with alcohols, the corresponding equilibrium can be reversed to produce the monomeric components by hydrolysis as shown in Fig. 7.2a. An alternative to the hydrolytic cleavage involves the alcoholysis with consequent production of esters (Fig. 7.2b), which are conveniently soluble in common organic solvents.

While in theory all the aforementioned reactions occur spontaneously depending on temperature and pressure, their efficiency can be increased adding a catalyst. Common catalysts for trans-esterification reactions consist of transition metal salts like the widely used zinc acetate. Based on such premises, in this study the methanolysis of a model URP compound as a function of the catalyst nature, as well as of the reaction temperature and duration, was successfully investigated.

In terms of new applications for the recovered NMF of PCBs, recent researches and patents demonstrated the feasibility of innovative emulsion technologies for the production of thermoplastic composites with high proportions of recycled fillers of various size and nature (organic and inorganic) [20, 21]. By applying this methodology, powders of fibre-reinforced polymers such as NMF of PCBs can be emulsified 
(a)

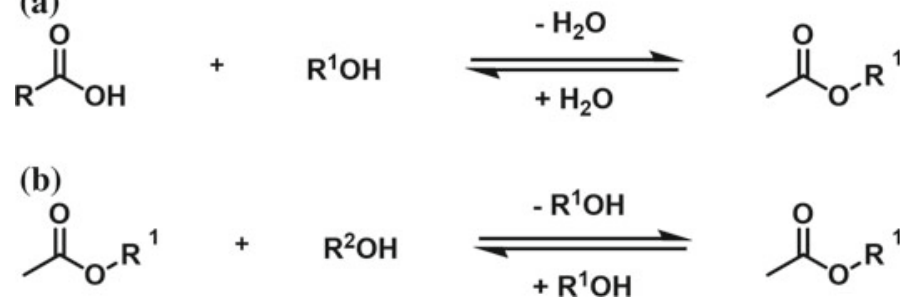

Fig. 7.2 Esterification-hydrolysis (a) and transesterification (b) reactions

and moulded in a thermoplastic matrix to obtain sheets or granules. In this study, this emulsification procedure was carried out at room temperature using a thermoplastic matrix made of expanded Polystyrene (EPS), which was preliminarily produced as a physical gel through a volatile organic solvent. Post-consumer packaging EPS is available in large quantities, since it is used as loose fill packaging for fragile products [22]. Moreover, its lightness makes its disposal highly difficult. The combination of these materials enabled to obtain low-cost recycled compounds with good performance thanks to the inclusion of a mixture of grinded PCBs and rubber tire into the EPS gel. In fact, EPS in the form of gel is able to combine a fluid state with the elasticity of the physical network. Such network results stable during subsequent machining operations, hence assuring good mechanical properties to the obtained composites.

\subsubsection{New Business Models for PCBs Recycling}

Multiple options are open for the uptake of smart PCBs pre-treatment processes and technologies (selective disassembly of PCBs components and optimised shredding and separation processes) by existing actors operating in the recycling chain or new companies. Existing literature was analysed [14] and a dedicated empirical analysis was carried in Italy to characterise typical recycling chains and to collect input for new business model design. Four treatment centres were visited and their management was interviewed to map the as-is situation and to ask their opinion on new technologies for efficient shredding and separation processes, which would substitute (or improve the performance) of current pyro-metallurgical processes. More precisely, the case study companies were an authorised consortium for the collection of WEEE, a company performing pyro-metallurgical processes on PCBs and two chemical companies recovering metals from PCBs ashes. Such a sample allowed to represent the whole recycling value chain, from PCBs collection to materials recovery. Based on this analysis, it can be argued that the main actors in the typical supply chain are: 


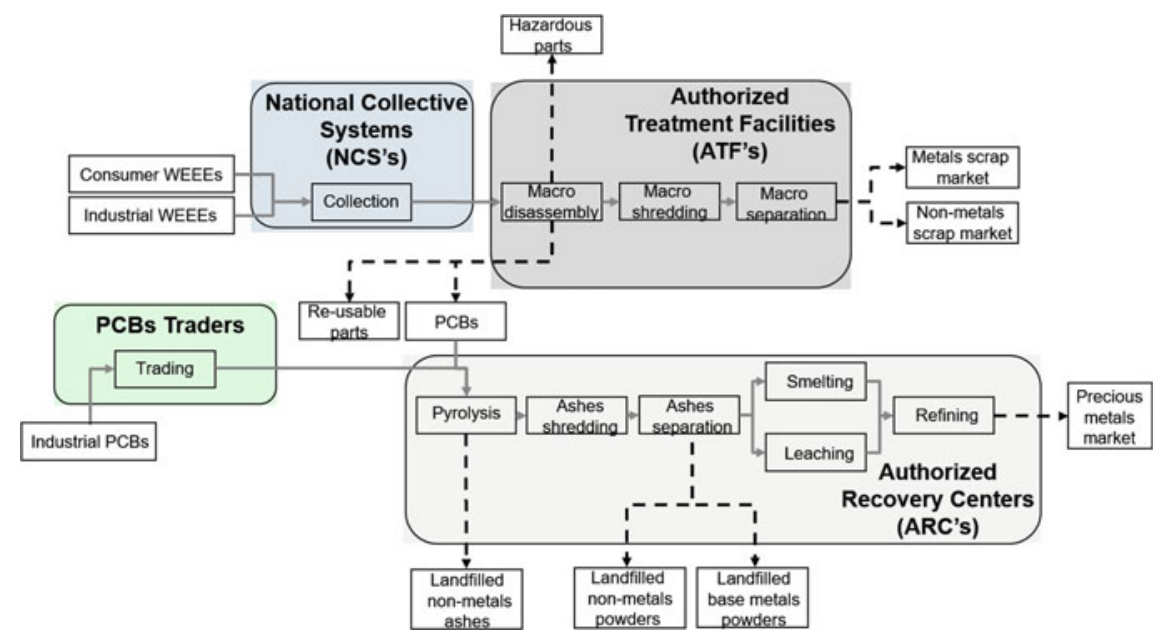

Fig. 7.3 Typical PCBs recycling chain

1. National Collective Systems that collect WEEE (NCSs). PCBs may also enter the recycling chain from specialised waste PCBs traders (Fig. 7.3).

2. Authorised Treatment Facilities (ATFs) that perform macro-disassembly of products and, eventually, macro-separation of scrap through rough shredding.

3. Authorised Recovery Centres (ARCs) that recover materials through chemical processes.

Three options were identified in this work for the uptake of novel PCBs recycling technologies, which would pave the way to new business models for supply chain actors. The first option is that new intelligent shredding and separation technologies, based on novel modelling and optimised control systems, are uptaken by new incoming actors, the Independent De-manufacturing Centres (IDCs). They will intercept PCBs flows coming from ATFs and scrap from PCBs traders, with the final aim to extract as much value as possible from them. Inside a hypothetical IDC, PCBs will be previously tested to identify valuable and reusable components that, in a following disassembly phase, will be separated and sold in the electronic components market. The remaining PCBs bare board will be sent to the shredding and separation phases. The resulting base metals and non-metals powders will be sold in their proper markets, while precious metals powders will be sent to ARCs for a further more detailed refining. This type of business model is the one seen as the most promising by the interviewed Italian actors in terms of possible implementation and profitability. From a scientific point of view, this type of business model could favour a high technology specialization and the development of knowledge around selective disassembly, shredding and separation processes. However, this option would offer lower proximity to the precious metals end-markets (managed by ARCs). It would also increase the exposure to PCBs collection fluctuations, with a high sourcing risk and contractual power of ATFs. 
The second option implies that ATFs add to their traditional process the new advanced processes and technologies for the treatment of PCBs, transforming themselves in Multi-treatment facilities (MTFs). MTFs could exploit their current knowledge and technologies to treat various types of waste products, among which PCBs. PCSs treatment through smart processes might increase the profitability of ATFs due to the high content of valuable materials of this new category of waste, which is added to the traditional waste products that ATFs already process. Compared to the scenario of IDCs, the uptake of new technologies would imply a lower risk, since ATFs are recycling companies already operating in the market and experienced in treatment technologies.

Finally, the third option of business model would imply that the ARCs add new smart pre-treatment technologies prior to their traditional chemical recovery processes, becoming Integrated Recovery Centres (IRCs). This scenario would generate a radical innovation in the recycling chain, because it would allow the integration of mechanical and chemical treatment at the premises of the same company, with the consequent optimization of process parameters of both phases. This business model would overcome the current fragmentation of the recycling chain and the local optimization approaches which do not consider the recycling process as a whole and the opportunity to efficiently recover both MF and NMF of PCBs. However, such a business model appears very challenging, since ARCs have traditional competence on chemical recovery processes but are culturally distant from mechanical processes. Thus, they might be averse to new technologies or might experience difficulties in the exploitation of their benefits.

\subsubsection{Prototypes}

Three industrial prototypes were developed based on the results of this work. The first one is a station for PCBs automatic components selective disassembly for reuse, remanufacturing or recycling (Fig. 7.4). Such a prototype demonstrates how pre-selected PCBs components can be disassembled in a totally automated way to preserve their residual functionalities or to avoid precious materials loss during the disassembly process. In the prototype, disassembly of PCBs components is based on a thermal reflow process guaranteeing high disassembly accuracy. In such a process, a reflow heater is used to heat the board and the components to melt the solder paste which anchors components on the board. Proper time-temperature profiles were identified to avoid overheating and damages to PCBs (still functioning) components. Based on such profiles, a structured control solution was realised to coordinate the machine actuators (hot air nozzle and bottom heater) in terms of heating power. To this aim, two coordinated control loops have been implemented and thermocouples have been installed and connected on both control loops. A Modbus/TCP has been also included to support the information exchange to/from the process supervision, i.e. list of disassembly operations to be performed and related parameters. 


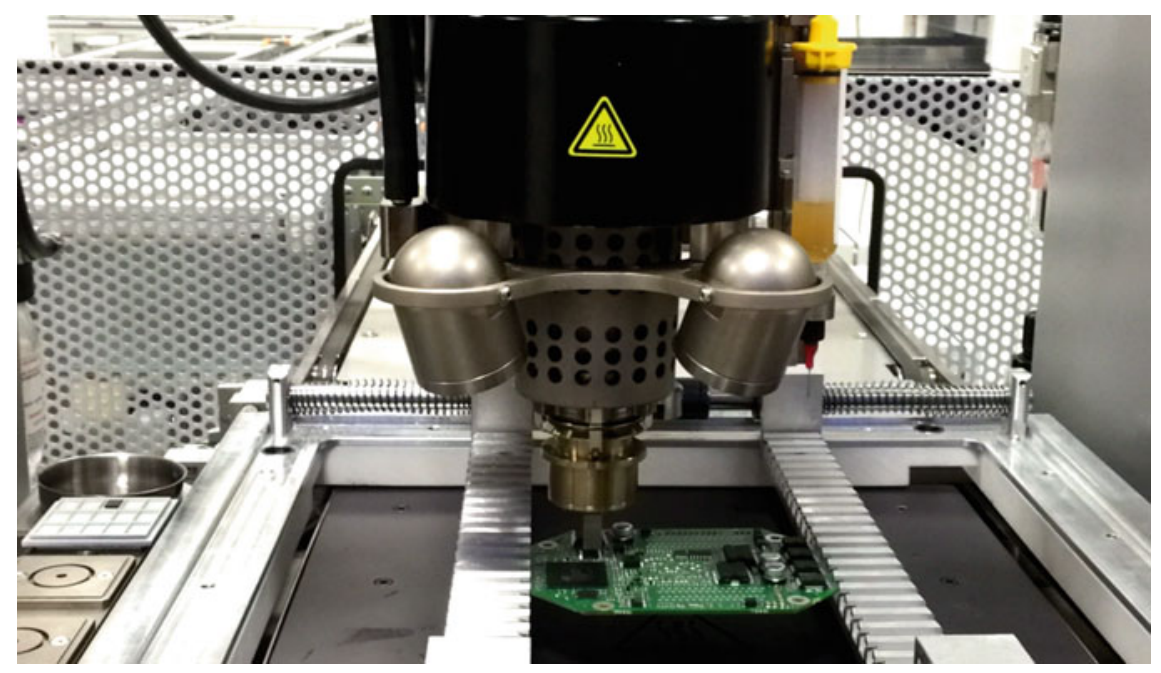

Fig. 7.4 Automatic PCB components selective disassembly prototype station

A linear motors-based gantry architecture has been adopted to guarantee tools positioning accuracy as well as proper dynamic capabilities. Moreover, a force control table has been integrated to properly control the contact force impressed by the pneumatic disassembly tools onto the PCB during operation in order to avoid reusable chips damage. In fact, the robot is moved under position control during normal operation. The force control loop is activated when the tool is in proximity of the PCB to be operated (about $5 \mathrm{~mm}$ far from the PCB surface). An automatic tool exchange system has been integrated in the machine architecture to guarantee machine flexibility in processing different electronic chip case shapes. Furthermore, a system for automatic load and unload of the PCB pallet has been included within the machine operation area.

A computer vision system was developed and integrated in the station to support on-line automatic recognition of the components to be processed. Such a system is capable of identifying the components by matching the PCB image acquired by the camera and the information related to the components' geometry stored in a repository. Such a repository includes the main classes of PCBs components in terms of family, dimensions and rework operation constraints (such as maximum temperatures and thermal gradients). The repository was built on purpose by accessing a large number of components' materials specifications through public catalogues of vendors and represents a relevant knowledge source that will need to be constantly updated in the future according to new PCBs technology and components. Once identified the component to be disassembled, its coordinates in the machine operation 
plan are automatically adapted and delivered to the station controller to automatically reconfigure the operation point. The vision system is also used to compensate possible errors in manipulating the PCB component by the gripper.

The second developed prototype is a system for the in-line prediction of PCBs material content and value (depending on materials' price), based on the recognition of PCB components. The hardware is composed of a linear camera for PCB image acquisition, a transportation system and a lighting system. In the system, the PCBs to be analysed are manually loaded on a conveyor and the linear camera mounted at optimised distance from the conveyor scans the PCB for image acquisition. The lighting system (LED) provides a diffuse-like illumination modality avoiding spots or highlights. The software tool is composed of two modules: the Image Processing Module and the Material Prediction Module. The first module includes several segmentation algorithms that use the image of PCB to identify the specific areas of PCBs representing the electronic components mounted on it. The general procedure requires a thresholding operation for the segmentation of both the black plastic and the metal terminations. Afterwards, threshold images are post-processed to select the objects of interest. In the case of black plastic, a modified watershed algorithm is used for the definition of the mass centre and the approximate shape of black plastic bodies. For metallic parts, a logical operation is performed with an innovative proximity algorithm to assign to each plastic body the set of relative metallic terminations. Finally, the segmented regions are classified into the different categories of electronics packages that were previously characterized through an extensive analysis of open source documentation (materials declarations). A probabilistic knowledgebased method is used, since the real image differs from the ideal situation because of the presence of non-interesting objects. This method provides better results in term of classification rate with respect to other standard methods such as pattern matching or artificial neural network. This is justified considering that the spatial intensity distribution around an electronic package is very similar for most of the classes of electronic packages, thus increasing the classification error.

The second software module consists in statistical models able to predict the material contents of electronic components as a function of the electronic package type, size and geometry. The complete algorithm has been tested using several PCBs scraps coming from different application field. During the experiments, the PCBs have been transported through the conveyor belt under the linear camera and an image for each PCB has been taken. The image has been processed with the algorithm of segmentation and classification. Figure 7.5 shows the PCBs used in the testing, while Fig. 7.6 shows the final result of image processing. The classification error amounted to $15 \%$.

Finally, the third prototype is a Cyber-Physical System (CPS) for a self-optimizing shredding stage to cope with the high variability of input products. In the prototype, coarse shredded particles undergo a Computerized Particle Analyser (CPA) for high speed granulometric analysis, which gives the dimensional distribution of the particles as output. The developed software tool acquires and stores this information, 


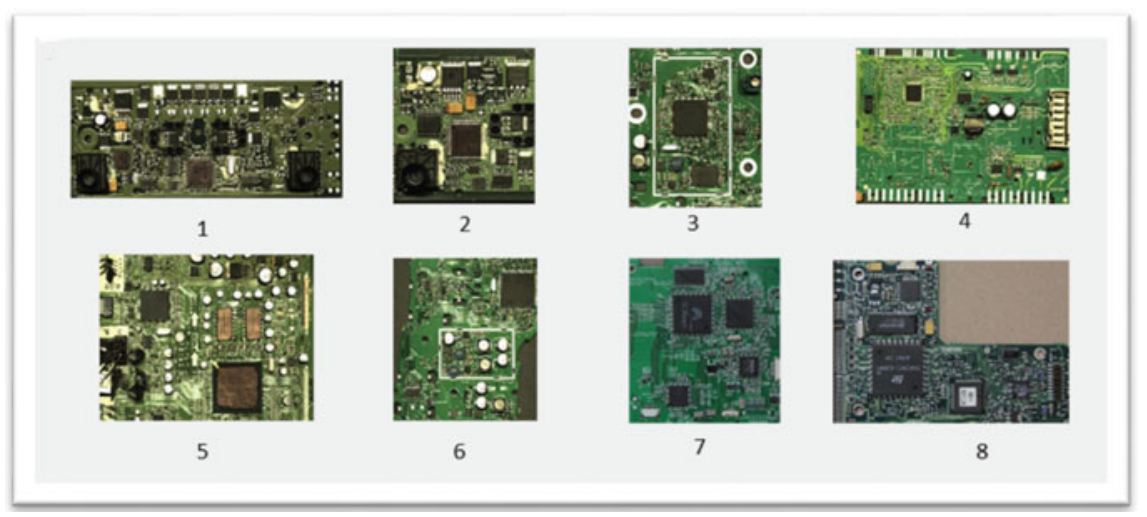

Fig. 7.5 PCBs used in prototype testing

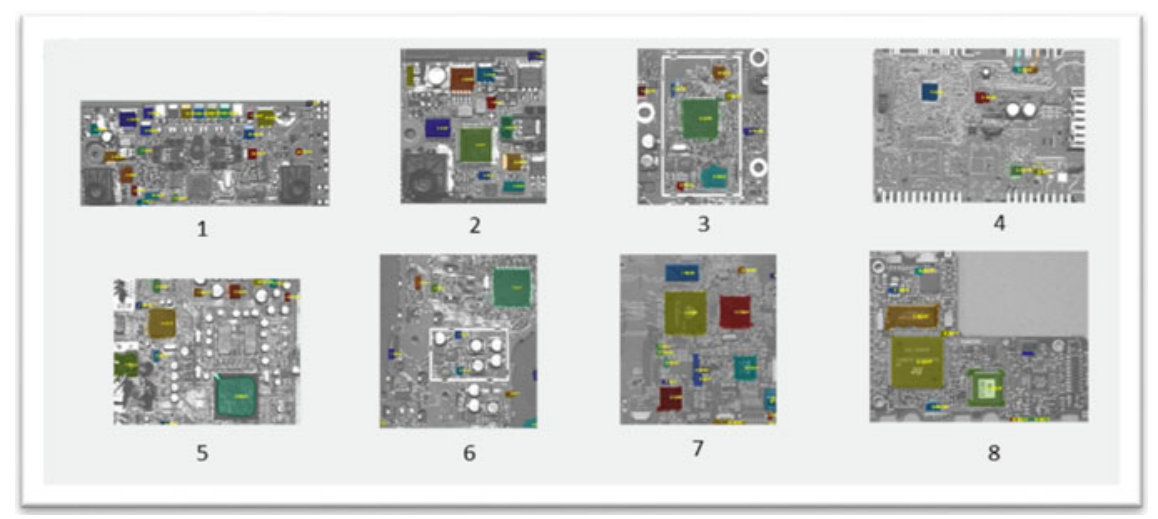

Fig. 7.6 Result of image processing

which is the input for a Population Balance Model (PBM) presented in Sect. 7.4.1. A human-machine interface was developed to acquire the target output distribution and throughput information from the operator. These data are processed by the optimization module that calculates and suggests (using least square method) the optimal process parameters to achieve targets. Meanwhile, particles are shredded by a fine comminution machine with optimal parameters and the output distribution is checked by the CPA before leaving the cyber-physical system. All the technologies have been integrated through a pneumatic transportation system. The overall prototype functioning is represented in Fig. 7.7. 


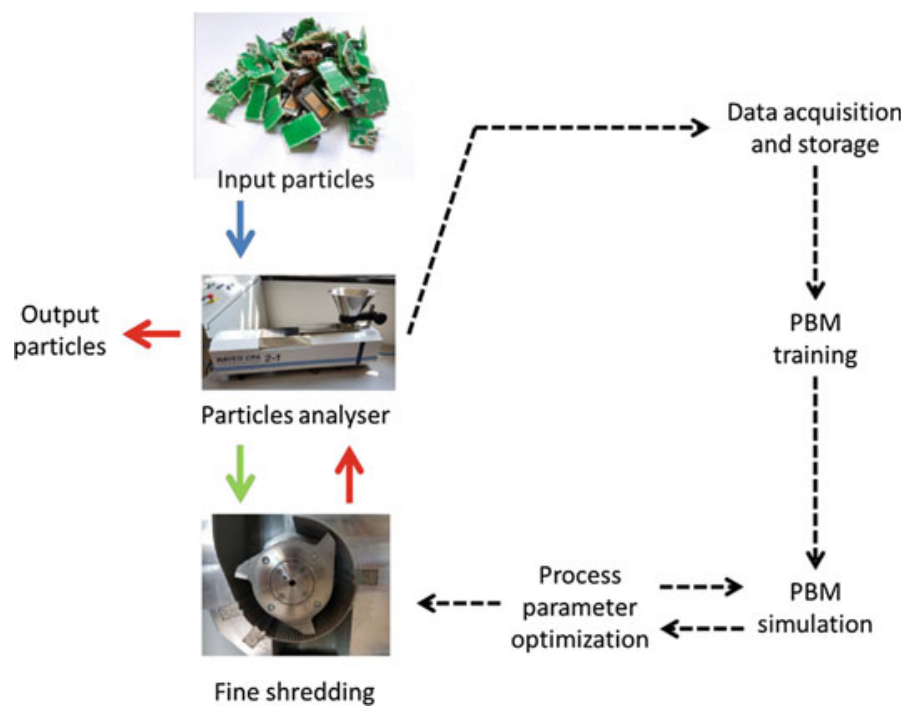

Fig. 7.7 CPS prototype functioning

\subsection{Industrial Testing and Results}

Technical solutions described in the previous section were tested and validated at laboratory scale. Results are described in the following subsections.

\subsubsection{Shredding Model Validation and Parameters Optimisation}

The experimental procedure mentioned in Sect. 7.4.1 was executed several times with input mixtures obtained from different types of PCBs (low, medium and high grade) coming from various sectors as automotive, white goods and personal computers, first for model training and then for model validation.

A relevant outcome for the rapid characterisation of the shredding machine, is that, within reasonable operational limits, the size distribution of output particles largely depends on the grate size and only weakly on the rotational speed. Therefore, this latter parameter can mainly be tuned to ensure an appropriate level of saturation of the comminution chamber for best performance depending on the input flow of material. Changing the speed will accelerate or slow down the process, affecting the throughput, but does not modify the output particles size distribution.

The validation experiments (both goodness-of-fit and out-of-sample validation) showed a good adhesion of predicted distribution to the real one. The weak dependence on rotational speed is a plausible assumption, as homogeneity and multiplica- 
tion hypothesis. In the stationary state, the particles size distribution is well approximated by the distribution predicted by the model. As a consequence, by exploiting the PBM simulation model, it is necessary to identify the discharging grate with the most suitable size to optimize a comminution process obtaining an output particles size distribution in the stationary state as similar as possible to a target one (determined by the needs of downstream material processing).

Shredding prototype validation and verification activities have shown an accurate output size distribution, with a Kolmogorov-Smirnov metric for the comparison between the predicted and the measured distributions lower than 0.072 , while the optimization time, defined as time to search for an optimal configuration of the controllable parameters, is almost instantaneous. Furthermore, the time to train the PBM breakage and selection function is below $120 \mathrm{~s}$ to obtain good results.

\subsubsection{Validation of HF-Free Process for MF Assessment}

The procedure developed for the assessment of the MF content in shredded PCBs was experimentally validated by mineralizing the same amount of powder from the same batch of shredded PCBs while using: (a) only $\mathrm{HNO}_{3}$ acid; (b) $\mathrm{HNO}_{3}$ acid and $\mathrm{HCl}$ acid in a ratio 1:3; (Aqua Regia); (c) $\mathrm{HNO}_{3}$ and Aqua Regia mixture with a 1:1 ratio. The ICP-MS (X Series, Thermo) was calibrated by using a multi elements rare earth standard solution (Inorganic Ventures) and precious metal standard solution (Inorganic Ventures). Preliminary results showed that the most suited method to mineralize the elements of interest was the Aqua Regia attack because with this acid attack it is possible to digest both lanthanides and precious metals. Using this method, only small amounts of insoluble materials (silica and plastics) were detected. After preliminary mineralization assays, the samples were mineralized following this procedure: about $0.3 \mathrm{~g}$ of samples were placed in a Teflon tube and Aqua Regia was introduced and left overnight prior to microwave digestion. Samples were subjected to microwave (ETHOS D, Milestone) irradiation at $250 \mathrm{~W}$ for $5 \mathrm{~min}, 500 \mathrm{~W}$ for $5 \mathrm{~min}, 650 \mathrm{~W}$ for $5 \mathrm{~min}$ in sealed vials. Before the acid attack, some powders were ball milled to evaluate if a lower grain size could promote a faster mineralization. Results showed that no difference was observed in mineralization time.

The cleavage reaction presented in Sect. 7.4.2 was experimentally tested to assess the chemical process for the obtainment of intermediate products suitable for further polymerization reactions from the organic fraction of PCBs. The choice of catalysts was based on a study about a transesterification reaction of dimethyl terephthalate in ethylene glycol catalysed by different acetates metals. As a preliminary screening, zinc acetate was used to identify the optimal conditions for experiments run at low temperature, e.g. refluxing methanol $\left(68^{\circ} \mathrm{C}\right)$. It must be stressed how the system was active even at a low temperature, considering that this reaction is commonly carried out in supercritical conditions. A brief metal salts screening has also been carried out, but zinc acetate turned out to be the most active. Following these encouraging results, a higher temperature screening was conducted in a jacketed reactor thermostated at 
Table 7.1 CPS prototype functioning

\begin{tabular}{l|l|l|l|l|l}
\hline $\mathrm{M}_{\mathrm{w}}(\mathrm{Da})$ & $\mathrm{M}_{\mathrm{n}}(\mathrm{Da})$ & $\mathrm{M}_{\mathrm{z}}(\mathrm{Da})$ & PDI & $\mathrm{M}_{\mathrm{z}} / \mathrm{M}_{\mathrm{w}}$ & $\mathrm{M}_{\mathrm{z}+1} / \mathrm{M}_{\mathrm{w}}$ \\
\hline 734 & 728 & 741 & 1.009 & 1.009 & 1.02 \\
\hline
\end{tabular}

$165^{\circ} \mathrm{C}$. Compared to the low temperature results, at a higher temperature the catalytic activity distribution resulted more narrow, with the cheap lead acetate being the best catalyst. For such reasons different lead salts were employed. Among the different counterions, the acetate salt still resulted to be the most active, as commonly reported in literature for zinc catalysed reactions.

Once defined the best catalytic conditions, the kinetic trend for this reaction was examined. Finally, the recovered depolymerised fraction was subjected to analysis by Gel Permeation Chromatography (GPS). The results of such analysis are reported in Table 7.1 and confirm, as expected, a monodispersed sample of low molecular weight which could be re-employed as a starting material for polymerisation.

Finally, the procedure to achieve new re-usable compounds from the NMF of PCBs was experimentally validated. EPS scraps were dissolved in an environmental low-impact solvent at room temperature obtaining a fluidified paste. Previously milled, PCB scraps with/without tire rubber powders (1:1 weight ratio with respect to EPS) were added. After mixing in a HAAKE Rheocord at room temperature, the obtained slurry was calendared between steel cylinders (distance $2 \mathrm{~mm}$ ) and immersed in cold water for $48 \mathrm{~h}$ to remove the solvent. Finally, dried sheets were pelletized and moulded using a heated press at $190{ }^{\circ} \mathrm{C}$ for $12 \mathrm{~min}$. The mechanical characterization performed on the samples has shown interesting properties of the prepared materials. In particular, flexural tests have shown that the compound containing only EPS + PCB is characterized by modulus and stress at the break values higher than the material containing also rubber tire scraps. The obtained mechanical properties are comparable to those of virgin PS and other technopolymers. On the contrary, samples containing also the rubber tire phase have shown higher resilience values evaluated by fracture tests, thus assessing that the presence of rubber component is able to significantly toughen the material.

\subsection{Conclusions and Future Research}

In this work, a set of technological and business enablers were proposed to address the challenge of PCBs recycling. Due to the complexity of this issue, a multi-disciplinary approach was taken to develop solutions impacting on various stages of the process, in a logic of supply chain integration. Such solutions support: the selective automated disassembly of valuable PCBs components for re-use or for the optimisation of the downstream shredding and separation processes; the optimisation of shredding through process modelling finalised to the definition of optimal parameters for the obtainment of a target mixture, depending on input waste material; the 
environmentally-friendly chemical characterisation of PCBs MF to analyse the content of precious materials in waste PCBs with high confidence; the recycling of PCBs NMF through a new cleavage chemical reaction to create re-usable compounds from organic powders and though a new application for the production of a composite material using compounds obtained from organic PCBs fraction, mixed with other fillers; the establishment of new recycling business models through the uptake of novel technological solutions by different supply chain actors.

Developed technical solutions were successfully implemented in industrial prototypes and tested in laboratory conditions. However, experimental conditions were limited because of the wide variety of possible PCBs and components technologies, as well as the business uncertainty that is typical of recycling business. In addition, the industrial applicability and sustainability of developed technologies and business models should be investigated more in detail. The following activities are outlined for future research and innovation:

- Evaluation of industrial feasibility of automatic selective disassembly process and identification of the product types that justify such an approach. This innovative process, in fact, is characterised by higher costs compared with low-cost manual disassembly. Thus, it is foreseen that such an application is justified only in the business cases where treated components are of high added-value. This can happen when they can be re-sold in the spare parts market or they are made of particularly valuable metals for which pre-concentration can guarantee higher efficiency of downstream separation processes and lower materials losses.

- Extend the validation of the shredding process model through a wider experimental campaign including different type of PCBs. Besides the validation and improvement of the model, other important activities deal with the estimation of the economic benefits of model-based process optimisation and the readiness of companies to accept such a sophisticated solution compared to the current way of operating shredding machines. From the research point of view, the integration between shredding and separation processes should be investigated in the future with a joint approach.

- Further testing of the robustness of the chemical methodologies developed to characterise PCBs MF and to allow recycling of PCBs NMF. In particular, for the characterisation process, the developed testing procedure should be applied to various samples of PBCs of different composition to test its performance. In addition, characterisation cost and lead time should be estimated and compared to industrial needs. Regarding PCBs NMF recycling, industrial and business sustainability should be assessed by identifying potential applications which makes it cost effective. Furthermore, Life Cycle Assessment analyses should be carried out to verify the effective environmental advantage of the recycling under the light of the additional operations that need to be introduced.

- Better characterisation of the proposed new business models through empirical research involving more companies at different levels of the supply chain and performing a quantitative assessment of economic and environmental performance at business model level, considering also risks associated with the uncertainty of 
recycling business. Detailed return on investment simulations should be elaborated for the adoption of the solutions realised in this work.

Acknowledgements This work has been funded by the Italian Ministry of Education, University and Research (MIUR) under the Flagship Project "Factories of the Future-Italy" (Progetto Bandiera "La Fabbrica del Futuro" [23], research projects "Zero Waste PCBs-Integrated Technological Solutions for Zero Waste Recycling of Printed Circuit Boards (PCBs)", "PCB-ID-In-line automated device for the identification of components and the characterization of materials and value in waste PCBs" and "ShredIT-Self-Optimizing Shredding Station for De-manufacturing Plants"). Authors are grateful to Marco Diani and Francesco Baiguera for their support to the finalisation of this research and publication.

\section{References}

1. Report on Critical raw materials for the EU, Report of the Ad-hoc Working Group on defining critical raw materials (2014) https://ec.europa.eu/docsroom/documents/10010/attachments/1/ translations/en/renditions/pdf. Accessed 30 July 2018

2. Copani G, Brusaferri A, Colledani M, Pedrocchi N, Sacco M, Tolio T (2012) Integrated demanufacturing systems as new approach to end-of-life management of mechatronic devices. In: Proceedings of the 10th global conference on sustainable manufacturing, Istanbul, 31 Oct-2 Nov 2012

3. Colledani M, Copani G, Rosa P (2014) Zero Waste PCBs: a new integrated solution for keymetals recovery from PCBs. In: Proceedings SUM 2014, second symposium on urban mining, Bergamo, Italy, 19-21 May 2014

4. Borrotti M, Pievatolo A, Critelli I, Degiorgi A, Colledani M (2015) A computer-aided methodology for the optimization of electrostatic separation processes in recycling. Appl Stoch Models Bus Ind 32(1):133-148

5. Colledani M, Copani G, Tolio T (2014) De-manufacturing systems. Procedia CIRP 17:14-19

6. Kara S, Pornprasitpol P, Kaebernick H (2006) Selective disassembly sequencing: a methodology for the end-of-life products. CIRP Ann 55(1):37-40

7. Hall WJ, Williams PT (2007) Processing waste printed circuit boards for material recovery. Circuit World 33(4):43-50

8. Cui J, Zhang L, (2008) Metallurgical recovery of metals from electronic waste: a review. J Hazard Mater 158(2, 3):228-256

9. Huang K, Xu Z (2009) Recycling of waste printed circuit boards: a review of current technologies and treatment status in China. J Hazard Mater 164:399-408

10. Goosey M, Kellner R (2002) A scoping study end-of-life printed circuit boards. Intellect Shipley Europe Limited. www.cfsd.org.uk/seeba/TD/reports/PCB_Study.pdf

11. Li J, Xu Z, Zhou Y (2007) Application of corona discharge and electrostatic force to separate metals and non-metals from crushed particles of waste printed circuit boards. J Electrost 65:233-238

12. Li J, Xu Z (2010) Environmentally friendly automatic line for recovering metal from waste printed circuit boards. Environ Sci Technol 44:1418-1423

13. Kulkarni P, Chellam S, Flanagan JB, Jayanty RKM (2007) Microwave digestion-ICP-MS for elemental analysis in ambient airborne fine particulate matter: rare earth elements and validation using a filter borne fine particle certified reference material. Anal Chim Acta 599:170-176

14. Ongondo FO, Williams ID, Cherrett TJ (2011) How are WEEE doing? A global review of management of electrical and electronic wastes. Waste Manag 31:714-730

15. Tolio T, Copani G, Terkaj W (2019) Key research priorities for factories of the future-part I: missions. In: Tolio T, Copani G, Terkaj W (eds) Factories of the future. Springer 
16. Colledani M, Tolio T (2013) Integrated process and system modelling for the design of material recycling systems. CIRP Ann 62(1):447-452

17. Tolio T, Copani G, Terkaj W (2019) Key research priorities for factories of the future-part II: pilot plants and funding mechanisms. In: Tolio T, Copani G, Terkaj W (eds) Factories of the future. Springer

18. Dehling HG, Gottschalk T, Hoffmann AC (2007). Stochastic modelling in process technology. Elsevier

19. Danadurai KSK, Chellam S, Lee CT, Fraser MP (2011) Trace elemental analysis of airborne particulate matter using dynamic reaction cell inductively coupled plasma-mass spectrometry: application to monitoring episodic industrial emission events. Anal Chim Acta 686:40-49

20. Cerruti P, Fedi F, Avolio R, Gentile G, Carfagna C, Persico P, Errico M, Malinconico M, Avella M (2014) Up-cycling end-of-use materials: highly filled thermoplastic composites obtained by loading waste carbon fiber composite into fluidified recycled polystyrene. Polym Compos 35(8):1621-1628

21. Avella M., Errico M, Fabozzi G, Lucchesi C, Lucchesi G, Malinconico M, Petrucci M, Ponzecchi E (2009) Process and plant for the production of composite thermoplastics and materials. International Patent WO/2009/072150

22. SUSRAC Report Summary, Cordis Europa. https://cordis.europa.eu/result/rcn/156514_en. html. Accessed 10 July 2018

23. Terkaj W, Tolio T (2019) The Italian flagship project: factories of the future. In: Tolio T, Copani G, Terkaj W (eds) Factories of the future. Springer

Open Access This book is licensed under the terms of the Creative Commons Attribution 4.0 International License (http://creativecommons.org/licenses/by/4.0/), which permits use, sharing, adaptation, distribution and reproduction in any medium or format, as long as you give appropriate credit to the original author(s) and the source, provide a link to the Creative Commons licence and indicate if changes were made.

The images or other third party material in this book are included in the book's Creative Commons licence, unless indicated otherwise in a credit line to the material. If material is not included in the book's Creative Commons licence and your intended use is not permitted by statutory regulation or exceeds the permitted use, you will need to obtain permission directly from the copyright holder. 